\title{
The complex combination of COVID-19 and diabetes: pleiotropic changes in glucose metabolism
}

\author{
Abdolkarim Mahrooz $\mathbb{D}^{1,2} \cdot$ Giovanna Muscogiuri $^{3} \cdot$ Raffaella Buzzetti $^{4} \cdot$ Ernesto Maddaloni $^{4}$
}

Received: 18 January 2021 / Accepted: 9 April 2021 / Published online: 22 April 2021

(C) The Author(s), under exclusive licence to Springer Science+Business Media, LLC, part of Springer Nature 2021

\begin{abstract}
Purpose Angiotensin converting enzyme 2 (ACE2) is the door for SARS-CoV-2, expressed in critical metabolic tissues. So, it is rational that the new virus causes pleiotropic alterations in glucose metabolism, resulting in the complication of preexisting diabetes's pathophysiology or creating new disease mechanisms. However, it seems that less attention has been paid to this issue. This review aimed to highlight the importance of long-term consequences and pleiotropic alterations in glucose metabolism following COVID-19 and emphasize the need for basic and clinical research in metabolism and endocrinology. Results SARS-CoV-2 shifts cellular metabolism from oxidative phosphorylation to glycolysis, which leads to a decrease in ATP generation. Together with metabolic imbalance, the impaired immune system elevates the susceptibility of patients with diabetes to this deadly virus. SARS-CoV-2-induced metabolic alterations in immune cells can result in hyper inflammation and a cytokine storm. Metabolic dysfunction may affect therapies against SARS-CoV-2 infection. The effective control of metabolic complications could prove useful therapeutic targets for combating COVID-19. It is also necessary to understand the long-term consequences that will affect patients with diabetes who survived COVID-19.

Conclusions Since the pathophysiology of COVID-19 is still mostly unknown, identifying the metabolic mechanisms contributing to its progression is essential to provide specific ways to prevent and improve this dangerous virus's detrimental effects. The findings show that the new virus may induce new-onset diabetes with uncertain metabolic and clinical features, supporting a potential role of COVID-19 in the development of diabetes.
\end{abstract}

Keywords COVID-19 $\cdot \mathrm{SARS}-\mathrm{CoV}-2 \cdot$ Diabetes $\cdot$ Glucose metabolism $\cdot$ Long-term consequences

\section{Introduction}

The 2019 novel coronavirus disease (COVID-19) pandemic, caused by severe acute respiratory syndrome coronavirus 2 (SARS-CoV-2), is seriously threatening health systems. As a global healthcare issue, this pandemic has posed a tremendous threat to humans and accounts for

Abdolkarim Mahrooz

amahrooz@mazums.ac.ir

1 Molecular and Cell Biology Research Center, Mazandaran University of Medical Sciences, Sari, Iran

2 Diabetes Research Center, Imam Khomeini Hospital, Mazandaran University of Medical Sciences, Sari, Iran

3 Sezione di Endocrinologia, Dipartimento di Medicina Clinica e Chirurgia, Università Federico II Napoli, Napoli, Italy

4 Department of Experimental Medicine, Sapienza University of Rome, Rome, Italy causing considerable morbidity and mortality worldwide. Diabetes is one of the most frequent comorbidity associated with COVID-19 [1], and the highest mortality rates have been registered in people with cardiometabolic disorders [2]. Reports from the Centers for Disease Control and Prevention (CDC) and other national health centers demonstrated that the risk of a fatal outcome from COVID19 could be up to $50 \%$ higher in diabetes patients than people without diabetes [3]. A meta-analysis conducted on six studies with 1527 patients showed that the incidence of diabetes was twofold greater in severe patients compared to their non-severe counterparts [3]. An elevated risk of severe complications, including Adult Respiratory Distress Syndrome and multiorgan failure, have been observed in patients with diabetes $[4,5]$. Also, COVID-19 patients with diabetes had an increased risk of mortality based on a recent meta-analysis $(\mathrm{OR}=2.21,95 \% \mathrm{CI}: 1.83-2.66, p<0.001)$ [6]. Furthermore, the role of systemic endothelial dysfunction in the pathophysiology of COVID-19 in patients with cardiometabolic disorders may be essential so that it may 
potentially be considered a new therapeutic target at minimizing the severity of this infection [7].

SARS-CoV-2 infection is accompanied by the release of many pro-inflammatory cytokines [tumor necrosis factor (TNF), interleukin-6 (IL-6), and IL-1 $\beta$ ] described as "cytokine storm", which correlates with vascular hyperpermeability, lung injury, multiorgan failure, and COVID-19 severity $[8,9]$. Increased inflammation and the massive production of cytokines can generate insulin resistance and also influence beta-cell function, involving a further reduction of insulin secretion [10]. This may induce hyperglycemia, which in turn can elevate the virulence of SARS-CoV-2 and reduces the phagocytic activity and polymorphonuclear leukocytes [4, 11]. Based on the findings of Zhu et al. [12], improved glycemic control had associated with better outcomes in COVID-19 patients and pre-existing T2D, so that virus people with well-controlled blood glucose (upper limit $\leq 10 \mathrm{mmol} / \mathrm{L}$ ) had markedly lower mortality in comparison to those with poorly controlled blood glucose (upper limit $>10 \mathrm{mmol} / \mathrm{L}$ ) during hospitalization.

On the other hand, it has also been suggested that COVID-19 may trigger diabetes onset in predisposed subjects. In this narrative review, we aim to describe this other side of the coin, discussing the possible effects of COVID-19 on glucose homeostasis and related mechanisms.

\section{Possible effects of COVID-19 on diabetes}

SARS-CoV-2 infection in individuals with diabetes may trigger stress and increase the release of hyperglycemic hormones, such as catecholamines and glucocorticoids, which would lead to increased blood glucose concentrations and abnormal glucose variability [13], causing an increased risk of metabolic emergencies. In particular, COVID-19 can elevate the risk of both diabetic ketoacidosis (DKA) and hyperosmolar hyperglycaemic state even in people without previously recognized diabetes [14, 15]. The risk of developing DKA is higher in people with T1D, and additional metabolic complications can occur in these patients [11, 15]. The importance of ketoacidosis and DKA can be prominent in children and adolescents. There is a significant increase in DKA and severe ketoacidosis at diabetes diagnosis in children and adolescents during the COVID-19 pandemic. More importantly, reduced medical services, fear of approaching the health care system, and more complex psychosocial factors may result in the delayed DKA diagnosis, which can be life-threatening [16]. Furthermore, more individuals with pre-existing type 2 diabetes (T2D) are progressing to insulin therapy with severe COVID-19 [14]. Maddaloni et al. first also hypothesized that in the long term, the infection of pancreatic beta-cells could trigger beta-cell autoimmunity [17], which might trigger type 1 diabetes (T1D) onset in predisposed subjects [18]. COVID-19 was associated with new and persistent hyperglycemia so that some individuals with normal HbA1C levels may develop new-onset diabetes [19]. However, the extent and phenotype of new-onset diabetes in patients with confirmed COVID-19 with a previous negative history of diabetes remains to be elucidated [11].

Some medications with a proven beneficial effect in COVID-19 and currently used for treating mild and severe COVID-19 cases, such as corticosteroids, can exacerbate glycemic control in patients with diabetes [11]. Although hyperglycemia is usually considered the primary concern in diabetes patients with COVID-19, hypoglycemic episodes resulting from drug use, viral pathogenesis, and metabolic perturbations of diabetes should not be ignored, particularly in older patients and in those prone to hypoglycemia [20, 21].

In addition to impaired glucose metabolism, diabetes is a chronic inflammatory disorder characterized by several vascular and metabolic abnormalities [22]. It is expected that SARS-CoV-2 infection could further worsen inflammation in individuals with diabetes. Together with metabolic imbalance, the impaired immune system elevates the susceptibility of patients with diabetes to pathogens such as SARS-CoV-2 [23]. Several factors involved in the immune dysfunction in diabetes patients, including hyperglycemia, impaired $\mathrm{T}$ cell function, impaired macrophage function, decreased neutrophil chemotaxis, and increased adherence of microorganisms to diabetic cells [23-27].

In T2D patients, an imbalance between coagulation and fibrinolysis carries out with increased coagulant potential, chronic platelet activation, and relative inhibition of fibrinolysis, predisposing the patients to the development of a hypercoagulable prothrombotic state [28]. This condition can be exacerbated in patients with COVID-19. Diabetes people with COVID-19 show a significantly higher D-dimer, a coagulation index, than those without diabetes. Notably, the risk of coagulopathy and thrombosis could be higher in diabetes and obesity, increasing susceptibility to thrombotic disorders [29]. Furthermore, based on some evidence, endothelial injury and coagulopathy may be central mediators of lung injury in SARSCoV-2 infection so that therapies that work on this basis may be considered for COVID-19-related ARDS [30].

Gestational diabetes mellitus is one of the most common medical problems found in pregnancy. Pregnant women with diabetes may be more vulnerable to the severe effects of SARS-CoV-2 infection [31, 32]. It seems that there are more clinical complications in pregnant women with COVID-19, and new infection was associated with enhanced preterm birth, preeclampsia, cesarean, and perinatal death [33]. Based on a case report study, pregnancy is a high-risk period for euglycemic ketoacidosis, even in nondiabetic women, particularly in infectious diseases such as 
COVID-19 [34]. Drug-induced hyperglycemia or secondary diabetes during COVID-19 treatment, particularly with frequent use of corticosteroids, maybe one of the causes of new-onset hyperglycemia in patients with SARS-CoV-2 infection. This can be observed especially in admitted patients with COVID-19 without a history of dysglycemia or diabetes and not on corticosteroids. New-onset diabetes in COVID-19 patients has been related to significantly higher complications and all-cause death than subjects with normoglycemia and preexisting diabetes [35].

\section{The long-term consequences of SARS-CoV-2 infection should be more attentioned, particularly in individuals with diabetes}

Although the long-term consequences for patients recovering from COVID-19 are unknown, researchers and healthcare professionals need to be aware of the importance of continued monitoring of long-lasting consequences in managing this strange disease. In this context, the results obtained for previous coronaviruses may be useful. Longterm outcomes following severe illness caused by SARS and MERS were analyzed in a meta-analysis of 28 studies. Accordingly, the increased prevalence of posttraumatic stress disorder, depression, and anxiety were reported up to 6 months after discharge [36]. The findings of a follow-up study for 12 years on 25 recovered SARS patients also showed that they tended to have increased hyperlipidemia, cardiovascular abnormality, and abnormal glucose metabolism, and significant differences found in serum metabolomes in comparison to healthy volunteers [37]. COVID19 survivors may, therefore, also experience long-lasting morbidity [38]. Based on study results, $87 \%$ of patients who had recovered from COVID-19 experienced persistence of at least one symptom (a mean of 60 days after onset of the first COVID-19 symptom), particularly dyspnea and fatigue [39]. However, it should be mentioned that adverse metabolic effects of SARS-CoV-2 infection may be permanent without specific clinical symptoms.

It is unknown at this time whether diabetes-related changes that occur in severe COVID-19 will disappear after the disease has resolved or will be permanent. Patients with severe COVID-19 could develop high degrees of insulin resistance that may be asymptomatic. This is consistent with the SARS virus findings, causing insulin resistance in $50 \%$ of the patients throughout infection [18]. Follow-up of the recovered patients with underlying metabolic disorders such as diabetes would be essential to evaluate developing microvascular and macrovascular complications in all the vascular beds, particularly the eye, kidney, and heart [40]. It is unknown how recovery from the infection does or does not differ from other forms of severe critical illness [30]. However, based on reports, sepsis recovery experiences may be applicable to patients recovering from severe COVID-19 [38]. Receipt of these practices has been related to lower odds of rehospitalization or death [38]. Taken together, it is noteworthy that post-recovery sequelae of COVID-19 may appear months and years after recovery, and it recommends that for many patients surviving COVID-19, health care should not end when they are discharged.

\section{The key role of ACE2 in the interaction between COVID-19 and diabetes}

The interaction between SARS-CoV-2 and angiotensinconverting enzyme 2 (ACE2) is an essential factor contributing to the new virus to develop different clinical and metabolic features. ACE2 is expressed in critical metabolic tissues, including pancreatic beta-cells, adipose tissue, liver, the small intestine, and the kidneys. It is rational that the new virus causes pleiotropic alterations in glucose metabolism, which would result in the complication of the pathophysiology of pre-existing diabetes or the creation of new mechanisms of disease [11].

ACE2 is the cellular receptor of SARS-CoV-2 and acts as the door for SARS-CoV-2 to enter host cells. The cellular serine protease TMPRSS2 is also needed for ACE2-mediated coronavirus entry into the cells [41]. The expression of ACE2 in different tissues is proportional to various symptoms of COVID-19, such as respiratory symptoms, which are predominant, acute cardiac and kidney injuries, gastrointestinal and liver function abnormalities, and beta-cell damage [4, 42]. Understanding the mechanisms of interaction between the renin-angiotensin system (RAS) and SARS-CoV-2 can provide adequate therapy for patients at an increased risk of COVID-19. ACE2 is a RAS member, which cleaves a single amino acid residue at the carboxyl terminus of Ang I (Ang 1-10) and Ang II (Ang 1-8) to form Ang 1-9 and Ang 1-7, respectively. Vasoconstrictive and inflammatory effects of Ang II are counterbalanced with Ang 1-7, which has vasodilatory and anti-inflammatory effects $[43,44]$. The endocytosis of SARS-CoV-2 results in a reduction in ACE2 activity and a shift from Ang 1-7 to Ang II. This alteration may lead to lung injury, as found in the SARS-CoV infection [45]. Therefore, it should be noted that ACE2 plays different roles related to COVID-19, so that it is the binding site for SARS-CoV-2, and on the other hand, its reduced expression by the virus may involve severe lung injury [44].

Moreover, a higher prevalence of hypokalemia due to renal potassium wasting is a clinical feature in patients with COVID-19, which can be explained by the downregulation of ACE2. This downregulation leads to decreased degradation of Ang II and, consequently, elevated aldosterone secretion [46]. Also, a decrease in angiotensin 1-7 and an 
increase in angiotensin II concentrations could lead to altered glucose metabolism in the virus's target cells.

According to a large phenome-wide Mendelian randomization study, T2D was causally associated with increased expression of ACE2 in the lung. Accordingly, elevated ACE2 expression due to diabetes and related traits may influence COVID-19 risk and exacerbate its complications [47]. A recent study's findings proposed that patients at old age, males, and those who have diseases associated with high expression of ACE2, including hypertension and diabetes, may have elevated risk for the delayed clearance of the virus [48]. It seems that the impacts of diabetes on ACE2 expression depend on the stage of the disease. According to experimental studies, ACE2 expression increases in early disease and decreases in later disease [49-51]. A study on mice showed that the lung's ACE2/ACE activity ratio significantly decreased in late-stage diabetes [52].

It is noteworthy that soluble ACE2 levels have significant associations with all metabolic syndrome components, including insulin resistance, obesity, hyperlipidemia, and hypertension. Intriguingly, APN01, as a recombinant soluble human ACE2, may have a protective role against acute lung injury and ARDS induced by SARS-CoV-2. This drug acts as a decoy receptor and prevents the virus from entering the cells. APN01 may be the most promising drug among all medications currently being developed [53]. It was suggested that the synthetic protease inhibitor camostat, which blocks TMPRSS2, may prevent and reverse hyperinsulinemia, hyperglycemia, and hyperlipidemia [54]. Moreover, the interaction between metformin and ACE2 can be important in COVID-19 infection. Metformin acts through AMP-activated protein kinase (AMPK) activation and can phosphorylate ACE2. This may result in conformational and functional alterations in the receptor, leading to the decreased binding of the receptor-binding domain of SARS-CoV-2 to ACE2 [55].

High and aberrantly glycated ACE2 in uncontrolled hyperglycemia may affect the binding of the spike protein to ACE2, so resulting in a higher propensity to COVID-19 and increased disease severity [56]. It should be noted that acute and chronic hyperglycemia could have different impacts on ACE2 expression. While acute hyperglycemia upregulates ACE2 expression facilitating viral cell entry, chronic hyperglycemia contributes to downregulating ACE2 expression, predisposing the cells to become vulnerable to the virus's inflammatory effects. Therefore, both chronic and acute hyperglycemia can have detrimental effects [4].

SARS-CoV-2 can also exert direct effects on beta-cells through ACE2, contributing to exacerbated outcomes in diabetes patients. The virus's entry into these cells may cause an acute beta-cell dysfunction that could lead to uncontrolled hyperglycemia [57, 58]. ACE2 was found in both islets and the exocrine glands. Strikingly, according to the GTEx database (https://gtexportal.org), the messenger RNA level of ACE2 was found to be higher in the pancreas compared with the lung [58], showing that the pancreas is an essential target of SARS-CoV-2. It was reported that $17 \%$ of severe COVID-19 patients had increased levels of amylase and lipase. The pancreas' focal enlargement or dilatation of the pancreatic duct was also present in $7.5 \%$ of patients with severe COVID-19 [58]. Direct beta-cell damage due to SARS-CoV-2 infection could theoretically cause insulin deficiency and autoantigen spread, leading to severe DKA, insulin dependency, and chronic pancreatic autoimmunity [4].

ACE2 is expressed on the arterial and venous endothelial cells of the adrenal glands [59]. It was also indicated that ACE2 and TMPRSS2 are colocalized in adrenocortical cells. The cortisol concentrations were found to be lower in critically ill patients with COVID-19 than non-COVID-19 critically ill patients [60]. People with adrenal insufficiency may have a higher rate of respiratory infection-related deaths, possibly due to impaired immune function and thus need further care during COVID-19 [59]. The adrenal glands contribute to the physiology of the stress response of the organism in health and disease that shows their important roles in the course of severe COVID-19 infection. During severe COVID-19, the adrenal glands can be seriously injured, characterized by the perivascular infiltration of CD3+ and CD8+ T-lymphocytes [61]. Furthermore, adrenal gland disorders such as hyperaldosteronism and adrenal insufficiency can result in carbohydrate metabolism dysregulation that can affect glucose homeostasis [62], and it should be given more attention in the COVID-19 pandemic.

\section{The importance of pleiotropic alterations in glucose metabolism}

Although SARS-CoV-2 infection is not primarily a metabolic disease, it is found that COVID-19 progression could be dependent on metabolic mechanisms. Critical conditions, such as COVID-19, significantly increase energy demands. SARS-CoV-2 must rewire cellular metabolism to produce macromolecules required for replication, assembly, and existence. Viral proteins elevate extracellular acidification, which is evidence for direct regulation of glucose metabolism by SARS-CoV-2 in addition to its transcriptional modulation [41]. Adenosine triphosphate (ATP) is generated through two related metabolic pathways, glycolysis, and the tricarboxylic acid (TCA) cycle (Krebs' cycle) [63]. The imbalance of glucose metabolism could be crucially contributing to respiratory pathogenic virus infection. Oxygen supplement at the earliest stage of COVID-19 was proposed to correct the unbalanced glucose aerobic 
metabolism [54]. It should be noted that metabolic dysfunction may affect therapies against SARS-CoV-2 infection because some drugs significantly depend on ATP to achieve functionality [64]. Moreover, medicines such as fenofibrate or cloperastine (FDA-approved drugs) that target host metabolic pathways could play a role in minimizing virus replication [41].

Like cancer cells, the metabolic milieu was rearranged in virus-infected cells to facilitate virus production and replication [65]. In general, viral infection in mammalian cells shifts cellular metabolism from oxidative phosphorylation to glycolysis, which leads to a decrease in ATP production [66]. Under an anaerobic condition in COVID-19 patients, on the one hand, the pyruvate produced from glucose during glycolysis was fermented to lactate, which leads to the generation of limited amounts of ATP for urgent biological needs. On the other hand, high replication of SARS-CoV-2 viruses in infected cells consumes many cellular ATP. As this condition persists (ATP depletion), lactate delivered to the liver failed to be metabolized through gluconeogenesis, causing increased blood lactate concentrations in COVID-19 patients [54].

Under the aerobic condition, glucose oxidation can also be performed through the pentose phosphate pathway in which nicotinamide adenine dinucleotide phosphate (NADPH) was produced. NADPH is required to maintain the proper ratio of oxidized glutathione to glutathione (GSH), which is crucial for the antioxidant defense system, contributing to tackling invasive pathogens microorganisms along with the immune system. Under the persistent hypoxia state, the depletion of GSH from the pentose phosphate pathway's blockage could induce oxidative damage during SARS-CoV-2 infection [54, 67]. Furthermore, the deficiency of glucose-6-phosphate dehydrogenase, the key enzyme in the pentose phosphate pathway, could result in cell apoptosis and inflammatory cytokine release noted in COVID-19 infection [68, 69].

Cellular glucose metabolism is hijacked in cells infected with the SARS-CoV-2 virus [70]. Pro-inflammatory cytokines activate immune cells such as macrophages and dendritic cells to change their metabolism to produce vast amounts of cytokines. In this metabolic reprogramming, these cells' ability to generate ATP switches from engaging mitochondrial oxidative phosphorylation to cytosolic glycolysis. Glycolysis allows pro-inflammatory activated immune cells to generate increased amounts of proinflammatory cytokines, including interleukins (IL- $1 \beta$, IL2 , IL-6, IL-8), interferons (IFN1 $\alpha$ and IFN-1 $\beta$ ), and TNF- $\alpha$ as well as chemokines. These agents can induce excessive amounts of reactive oxygen and reactive nitrogen species resulting in extensive nitro-oxidative stress, which involves respiratory pathophysiology [70, 71]. Targeting cytokines and effectively suppressing the cytokine storm during
SARS-CoV-2 infection could help prevent the deterioration of COVID-19 patients and reduce mortality [9, 72]. However, it should be noted that therapeutic strategies for targeting the overactive cytokine response must be balanced with maintaining an adequate inflammatory response to clear the virus [8].

Melatonin forces activated immune cells to abandon glycolysis in favor of mitochondrial oxidative phosphorylation [70]. Furthermore, this hormone induces Bmal1, a circadian gene, which inhibits pyruvate dehydrogenase kinase (PDK) and leads to the disinhibition of the pyruvate dehydrogenase complex (PDC). PDC catalyzes the mitochondrial conversion of pyruvate to acetyl-coenzyme A, thereby elevating the TCA cycle, oxidative phosphorylation, and ultimately an increase in ATP production [73]. Intriguingly, to counteract quarantine-related sleep disorders, it was recommended to use food containing or promoting serotonin and melatonin synthesis at dinner such as leaves, roots, fruits, and seeds such as almonds, cherries, bananas, and oats along with increased physical activity [74].

PDC links glycolysis to TCA and fatty acid synthesis and can be a crucial modulator of energy and metabolic homeostasis. Ang II downregulates the activity of PDC through its phosphorylation and acetylation. The reduced PDC can decrease the rate of glycolysis, leading to an uncoupling between glycolysis and TCA, which would induce intracellular acidosis and energy depletion [66]. Insulin resistance is related to elevated skeletal muscle PDK contributing to the phosphorylation and inactivation of PDC. It is noteworthy that dichloroacetate, as a PDK4 inhibitor, could restore PDC activity and ATP level, improve metabolism disorders, suppress cytokine storm and viral replication [75].

The key rate-limiting enzymes in glycolysis, including pyruvate kinase isozyme and hexokinase 2 , were upregulated in SARS-CoV-2 infection [41]. The conversion of phosphoenolpyruvate to pyruvate is a crucial step in glycolysis, which is catalyzed by PK. PKM1 and PKM2 are the two major isoenzymes of PK. PKM1 is the more enzymatically active form and contributes to the entry of pyruvate into TCA. PKM2 exhibits lower enzymatic activity than PKM1 and involves elevated glycolysis, leading to the cytosolic accumulation of lactate and other metabolic intermediates [63]. Cytosolic lactate levels and lactate to pyruvate ratio were increased in COVID-19 patients requiring ICU admission compared with healthy controls, showing that the increase in lactate can be due to a fundamental metabolic shift rather than merely an enhancement in overall metabolism [63]. It was also reported that lactate dehydrogenase levels were moderately higher in the deceased than in survival elderly patients with COVID-19 [76].

Inactivated immune cells, decreased TCA could lead to the accumulation of several metabolites, including 
succinate, which is a crucial TCA cycle intermediate. As an endogenous danger signal, Succinate contributes to stabilizing hypoxia-inducible factor-1a, which increases the expression of IL-1ß during inflammation [76]. It was indicated that the dysregulated TCA and the upregulated glycolysis were mediated by the inflammatory transcription factors NF- $\mathrm{KB}$ and RELA [41]. In a multi-center study on elderly patients with COVID-19, immune-related metabolic index (an index based on the flux of corresponding metabolic reactions) was found as a potential risk factor (odds ratio: 6.42 [95\% CI 2.66-15.48], $p<0.001$ ) [76].

After entering the human cells, SARS-CoV-2 increases the hexosamine biosynthetic pathway (HBP) to secure excessive glucose consumption and substrates for rapid replication. This enhanced HBP would result in an excess of the enzyme O-GlcNAc (N-acetylglucosamine) transferase (OGT), which would, in turn, trigger massive amounts of interferon regulatory factor-5 (IRF5). IRF5 plays a role as a mediator in the induction of proinflammatory cytokines such as IL-6, IL-12, IL-23, and TNF- $\alpha$, is contributed to the recruitment of inflammatory genes with NF-KB and in determining the inflammatory macrophage phenotype. IRF5 and OGT contribute to worsening the IкB kinase-NF-кB pro-inflammatory pathway resulting in a tremendous inflammatory cytokine gene overexpression profile and deleterious endoplasmic reticulum stress that ultimately lead to hyperinflammation, a cytokine storm, and multiorgan failure (Fig. 1) [65]. Furthermore, SARS-CoV-2, through this mechanism, may increase vascular complications in patients with diabetes, and it may also explain the sudden cardiac deaths in COVID-19 [77].
Fig. 1 SARS-CoV-2-induced alterations in glucose metabolism. In mammalian cells, SARS-CoV-2 shifts cellular metabolism from oxidative phosphorylation to glycolysis, which results in a decrease in ATP generation. The key rate-limiting enzymes in glycolysis, including PKM2 and HK2, were upregulated in COVID-19. The decreased PDC can lead to an uncoupling between glycolysis and TCA, which would induce intracellular acidosis and energy depletion. Under an anaerobic condition in COVID-19, the depletion of GSH from the pentose phosphate pathway's blockage could cause oxidative damage by SARS-CoV-2. The new virus elevates the hexosamine biosynthetic pathway, which would result in massive amounts of IRF5. IRF5 involves worsening pro-inflammatory pathways that ultimately lead to hyper inflammation, a cytokine storm, and multiorgan failure. GSH, glutathione; HK2, hexokinase 2; IRF5, interferon regulatory factor-5; $\mathrm{LDH}$, lactate dehydrogenase; NADPH, nicotinamide adenine dinucleotide phosphate; PDC, pyruvate dehydrogenase complex; PEP, phosphoenolpyruvate; PDK, pyruvate dehydrogenase kinase; PKM2, pyruvate kinase isozyme M2; TCA, tricarboxylic acid
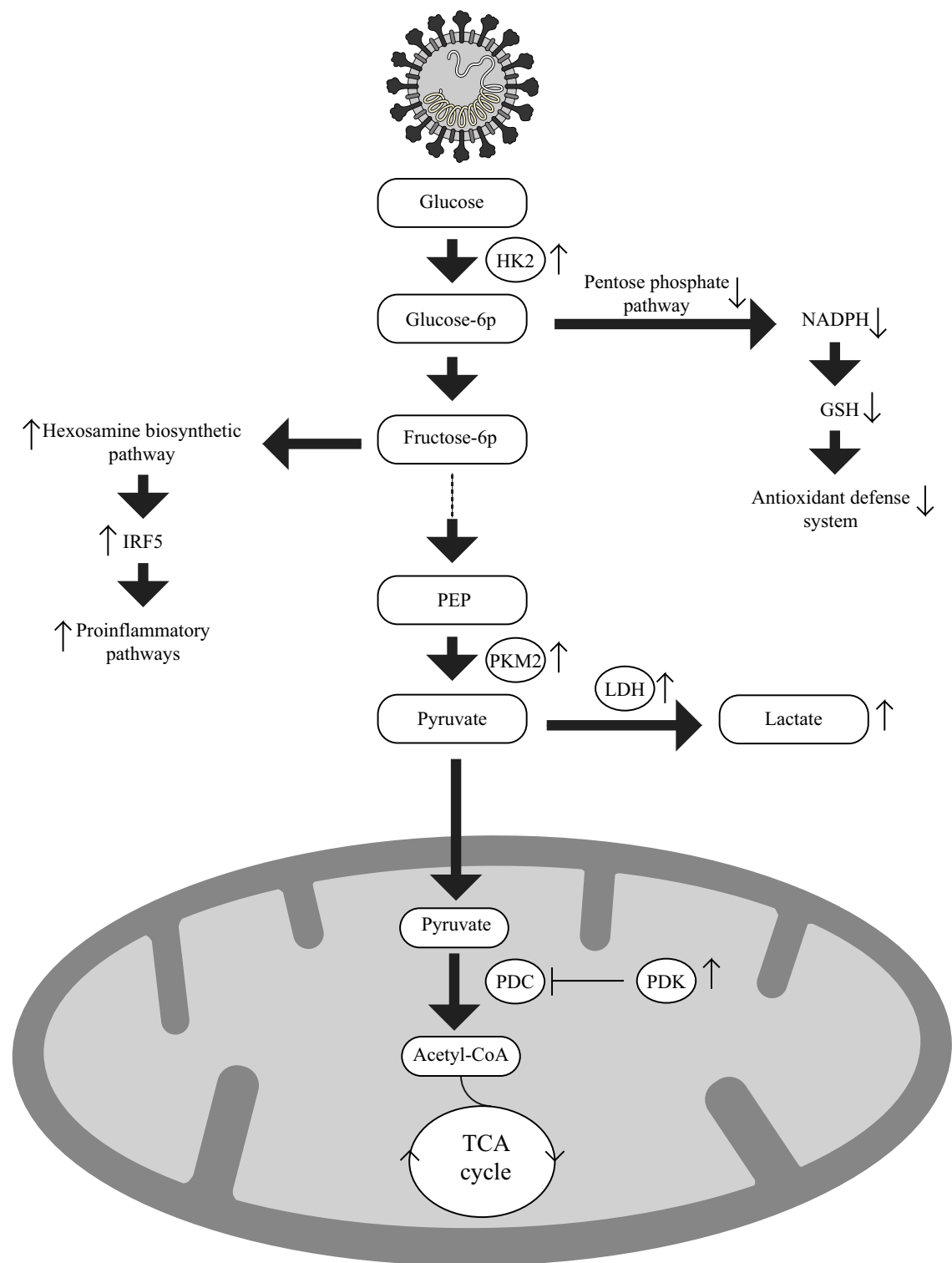


\section{Conclusion}

Since the pathophysiology of COVID-19 is still mostly unknown, identifying the metabolic mechanisms contributing to its progression is essential to provide specific ways to prevent and improve this dangerous virus's detrimental effects. It is also necessary to understand the long-term consequences that will affect the health of patients with diabetes who survived COVID-19. SARS-CoV-2-induced metabolic alterations in immune cells can result in hyper inflammation and a cytokine storm. These alterations could prove useful therapeutic targets for combating COVID-19. The findings show that the new virus may induce new-onset diabetes with uncertain metabolic and clinical features, supporting a potential role of COVID-19 in the development of diabetes.

\section{Compliance with ethical standards}

Conflict of interest The authors declare no competing interests.

Publisher's note Springer Nature remains neutral with regard to jurisdictional claims in published maps and institutional affiliations.

\section{References}

1. C. Covid, C. COVID, C. COVID, N. Chow, K. Fleming-Dutra, R. Gierke et al. Preliminary estimates of the prevalence of selected underlying health conditions among patients with coronavirus disease 2019-United States, February 12-March 28, 2020. Morbidity Mortal. Wkly. Rep. 69(13), 382 (2020)

2. E. Maddaloni, L. D’Onofrio, F. Alessandri, C. Mignogna, G. Leto, G. Pascarella et al. Cardiometabolic multimorbidity is associated with a worse Covid-19 prognosis than individual cardiometabolic risk factors: a multicentre retrospective study (CoViDiab II). Cardiovasc. Diabetol. 19(1), 1-11 (2020)

3. B. Li, J. Yang, F. Zhao, L. Zhi, X. Wang, L. Liu et al. Prevalence and impact of cardiovascular metabolic diseases on COVID-19 in China. Clin. Res. Cardiol. 109(5), 531-538 (2020)

4. S.R. Bornstein, F. Rubino, K. Khunti, G. Mingrone, D. Hopkins, A.L. Birkenfeld et al. Practical recommendations for the management of diabetes in patients with COVID-19. Lancet Diabetes Endocrinol. 8, 546-550 (2020)

5. S. Mirmohammadi, A. Kianmehr, M. Arefi, A. Mahrooz, Biochemical parameters and pathogenesis of SARS-CoV-2 infection in vital organs: COVID-19 outbreak in Iran. N. Microbes N. Infect. 38, $100792(2020)$

6. L. Shang, M. Shao, Q. Guo, J. Shi, Y. Zhao, J. Xiaokereti et al. Diabetes mellitus is associated with severe infection and mortality in patients with COVID-19: a systematic review and metaanalysis. Arch. Med. Res. 51, 700-709 (2020)

7. A. De Lorenzo, S. Escobar, E. Tibiriçá, Systemic endothelial dysfunction: a common pathway for COVID-19, cardiovascular and metabolic diseases. Nutr., Metab. Cardiovasc. Dis. 30(8), 1401-1402 (2020)

8. R.J. Jose, A. Manuel, COVID-19 cytokine storm: the interplay between inflammation and coagulation. Lancet Respir. Med. 8(6), e46-e47 (2020)
9. D. Ragab, H. Salah Eldin, M. Taeimah, R. Khattab, R. Salem, The COVID-19 cytokine storm; what we know so far. Front. Immunol. 11, $1446(2020)$

10. A. Ceriello, V. De Nigris, F. Prattichizzo, Why is hyperglycaemia worsening COVID-19 and its prognosis? Diabetes, Obes. Metab. 22(10), 1951-1952 (2020)

11. S. Cuschieri, S. Grech, COVID-19 and diabetes: the why, the what and the how. J. Diabetes Complicat. 34, 107637 (2020)

12. L. Zhu, Z.-G. She, X. Cheng, J.-J. Qin, X.-J. Zhang, J. Cai et al. Association of blood glucose control and outcomes in patients with COVID-19 and pre-existing type 2 diabetes. Cell Metab. 31, 1068-1077 (2020)

13. A. Wang, W. Zhao, Z. Xu, J. Gu, Timely blood glucose management for the outbreak of 2019 novel coronavirus disease (COVID-19) is urgently needed. Diabetes Res. Clin. Pract. 162, 108118 (2020)

14. England N., Improvement N. After-care needs of inpatients recovering from COVID-19. 2020;001559

15. J. Li, X. Wang, J. Chen, X. Zuo, H. Zhang, A. Deng, COVID-19 infection may cause ketosis and ketoacidosis. Diabetes, Obes. Metab. 22, 1935-1941 (2020)

16. C. Kamrath, K. Mönkemöller, T. Biester, T.R. Rohrer, K. Warncke, J. Hammersen et al. Ketoacidosis in children and adolescents with newly diagnosed type 1 diabetes during the COVID19 pandemic in Germany. JAMA 324, 801-804 (2020)

17. E. Maddaloni, R. Buzzetti, Covid-19 and diabetes mellitus: unveiling the interaction of two pandemics. Diabetes/Metab. Res. Rev 36, e33213321 (2020)

18. L. Marchand, M. Pecquet, C. Luyton, Type 1 diabetes onset triggered by COVID-19. Acta Diabetologica 57, 1265-1266 (2020)

19. S. Smith, A. Boppana, J. A. Traupman, E. Unson, D. A. Maddock, K. Y. Chao, et al. Impaired glucose metabolism in patients with diabetes, prediabetes and obesity is associated with severe Covid19. J. Med. Virol. 93, 409-415 (2021)

20. A. Hussain, B. Bhowmik, N.C. do Vale Moreira, COVID-19 and diabetes: Knowledge in progress. Diabetes Res. Clin. Pract. 162, $108142(2020)$

21. A. Yehya, S. Carbone, Managing type 2 diabetes mellitus during COVID-19 pandemic: the bittersweet. Diabetes/Metab Res Rev 37, e3360 (2021)

22. S. Knapp, Diabetes and infection: Is there a link?-A minireview. Gerontology 59(2), 99-104 (2013)

23. G. Daryabor, M.R. Atashzar, D. Kabelitz, S. Meri, K. Kalantar, The effects of type 2 diabetes mellitus on organ metabolism and the immune system. Front. Immunol. 11, 1582 (2020)

24. M.I. Maiorino, G. Bellastella, D. Giugliano, K. Esposito, Cooling down inflammation in type 2 diabetes: how strong is the evidence for cardiometabolic benefit? Endocrine 55(2), 360-365 (2017)

25. A. Berbudi, N. Rahmadika, A. Cahyadi, R. Ruslami, Type 2 Diabetes and its Impact on the Immune System. Curr. diabetes Rev. 16(5), 442 (2020)

26. M. Delamaire, D. Maugendre, M. Moreno, M.C. Le Goff, H. Allannic, B. Genetet, Impaired leucocyte functions in diabetic patients. Diabet. Med. 14(1), 29-34 (1997)

27. S.N. Zykova, T.G. Jenssen, M. Berdal, R. Olsen, R. Myklebust, R. Seljelid, Altered cytokine and nitric oxide secretion in vitro by macrophages from diabetic type II-like $\mathrm{db} / \mathrm{db}$ mice. Diabetes 49 (9), 1451-1458 (2000)

28. G. Patti, I. Cavallari, F. Andreotti, P. Calabro, P. Cirillo, G. Denas et al. Prevention of atherothrombotic events in patients with diabetes mellitus: from antithrombotic therapies to new-generation glucose-lowering drugs. Nat. Rev. Cardiol. 16(2), 113-130 (2019)

29. W. Guo, M. Li, Y. Dong, H. Zhou, Z. Zhang, C. Tian, et al. Diabetes is a risk factor for the progression and prognosis of COVID-19. Diabetes/Metab. Res. Rev 36, e3319 (2020) 
30. L.D.J. Bos, D. Brodie, C.S. Calfee, Severe COVID-19 infections -knowledge gained and remaining questions. JAMA Intern. Med. 181, 9-11 (2021)

31. H.R. Murphy, Managing diabetes in pregnancy before, during, and after COVID-19. Diabetes Technol. Ther. 22(6), 454-461 (2020)

32. T.E. van Gemert, R.G. Moses, A.V. Pape, G.J. Morris, Gestational diabetes mellitus testing in the COVID-19 pandemic: the problems with simplifying the diagnostic process. Aust. N.Z. J. Obstet. Gynaecol. 60(5), 671-674 (2020)

33. D. Di Mascio, A. Khalil, G. Saccone, G. Rizzo, D. Buca, M. Liberati et al. Outcome of coronavirus spectrum infections (SARS, MERS, COVID-19) during pregnancy: a systematic review and meta-analysis. Am. J. Obstet. Gynecol. 2(2), 100107 (2020)

34. S. Smati, P. M. Moreau, A. Bourdiol, S. Ploteau, S. Hadjadj, B. Cariou, Euglycaemic ketoacidosis during gestational diabetes with concomitant COVID-19 infection. Diabetes Metab. 47, 101181 (2021)

35. A.K. Singh, R. Singh, Hyperglycemia without diabetes and newonset diabetes are both associated with poorer outcomes in COVID-19. Diabetes Res. Clin. Pract. 167, 108382 (2020)

36. J. Zhou, J. Tan, Diabetes patients with COVID-19 need better blood glucose management in Wuhan, China. Metabolism 107, 154216 (2020)

37. Q. Wu, L. Zhou, X. Sun, Z. Yan, C. Hu, J. Wu et al. Altered lipid metabolism in recovered SARS patients twelve years after infection. Sci. Rep. 7(1), 1-12 (2017)

38. H.C. Prescott, T.D. Girard, Recovery From Severe COVID-19: leveraging the Lessons of Survival From Sepsis. JAMA 324(8), 739-740 (2020)

39. A. Carfi, R. Bernabei, F. Landi, Persistent symptoms in patients after acute COVID-19. JAMA 324, 603-605 (2020)

40. R. Dalan, B. O. Boehm, The implications of COVID-19 infection on the endothelium: a metabolic vascular perspective. Diabetes/ Metab. Res. Rev 37, e3402 (2021)

41. Ehrlich A., Uhl S., Ioannidis K., Hofree M., tenOever B. R., Nahmias Y. The SARS-CoV-2 transcriptional metabolic signature in lung epithelium. Available at SSRN 3650499

42. M. Gheblawi, K. Wang, A. Viveiros, Q. Nguyen, J.-C. Zhong, A. J. Turner et al. Angiotensin-converting enzyme 2: SARS-CoV-2 receptor and regulator of the renin-angiotensin system: celebrating the 20th anniversary of the discovery of ACE2. Circ. Res. 126 (10), 1456-1474 (2020)

43. C.M. Ferrario, A.J. Trask, J.A. Jessup, Advances in biochemical and functional roles of angiotensin-converting enzyme 2 and angiotensin-(1-7) in regulation of cardiovascular function. Am. J. Physiol.-Heart Circulatory Physiol. 289(6), H2281-H2290 (2005)

44. M. AlGhatrif, O. Cingolani, E.G. Lakatta, The dilemma of coronavirus disease 2019, aging, and cardiovascular disease: insights from cardiovascular aging science. JAMA Cardiol. 5, 747-748 (2020)

45. K. Kuba, Y. Imai, S. Rao, H. Gao, F. Guo, B. Guan et al. A crucial role of angiotensin converting enzyme 2 (ACE2) in SARS coronavirus-induced lung injury. Nat. Med. 11(8), 875-879 (2005)

46. Li X., Hu C., Su F., Dai J. Hypokalemia and clinical implications in patients with coronavirus disease 2019 (COVID-19). MedRxiv. 2020

47. S. Rao, A. Lau, H.-C. So, Exploring diseases/traits and blood proteins causally related to expression of ACE2, the putative receptor of SARS-CoV-2: A Mendelian Randomization analysis highlights tentative relevance of diabetes-related traits. Diabetes Care. 43, 1416-1426 (2020)

48. Chen X., Hu W., Ling J., Mo P., Zhang Y., Jiang Q., et al. Hypertension and diabetes delay the viral clearance in COVID-19 patients. medRxiv. 2020
49. S.M. Bindom, E. Lazartigues, The sweeter side of ACE2: physiological evidence for a role in diabetes. Mol. Cell. Endocrinol. 302(2), 193-202 (2009)

50. M. Ye, J. Wysocki, P. Naaz, M.R. Salabat, M.S. LaPointe, D. Batlle, A.C.E. Increased, 2 and decreased ACE protein in renal tubules from diabetic mice: a renoprotective combination? Hypertension 43(5), 1120-1125 (2004)

51. J. Wysocki, M. Ye, M.J. Soler, S.B. Gurley, H.D. Xiao, K.E. Bernstein et al. ACE and ACE2 activity in diabetic mice. Diabetes 55(7), 2132-2139 (2006)

52. H. Roca-Ho, M. Riera, V. Palau, J. Pascual, M.J. Soler, Characterization of ACE and ACE2 expression within different organs of the NOD mouse. Int. J. Mol. Sci. 18(3), 563 (2017)

53. A.M. Angelidi, M.J. Belanger, C.S. Mantzoros, Commentary: COVID-19 and diabetes mellitus: what we know, how our patients should be treated now, and what should happen next. Metabolism 107, 154245 (2020)

54. Z. Li, G. Liu, L. Wang, Y. Liang, Q. Zhou, F. Wu et al. From the insight of glucose metabolism disorder: Oxygen therapy and blood glucose monitoring are crucial for quarantined COVID-19 patients. Ecotoxicol. Environ. Saf. 197, 110614 (2020)

55. S. Sharma, A. Ray, B. Sadasivam, Metformin in COVID-19: a possible role beyond diabetes. Diabetes Res. Clin. Pract. 164, 108183 (2020)

56. A. Brufsky, Hyperglycemia, hydroxychloroquine, and the COVID-19 pandemic. J. Med. Virol. 92(7), 770-775 (2020)

57. G. Lisco, A. De Tullio, V.A. Giagulli, E. Guastamacchia, G. De Pergola, V. Triggiani, Hypothesized mechanisms explaining poor prognosis in type 2 diabetes patients with COVID-19: a review. Endocrine 70, 441-453 (2020)

58. F. Liu, X. Long, B. Zhang, W. Zhang, X. Chen, Z. Zhang, ACE2 expression in pancreas may cause pancreatic damage after SARSCoV-2 infection. Clin. Gastroenterol. Hepatol. 18(9), 2128-2130 (2020)

59. R. Pal, COVID-19, hypothalamo-pituitary-adrenal axis and clinical implications. Endocrine 68(2), 251-252 (2020)

60. Y. Mao, B. Xu, W. Guan, D. Xu, F. Li, R. Ren et al. The adrenal cortex, an underestimated site of SARS-CoV-2 infection. Front. Endocrinol. 11, 593179 (2020)

61. V.A. Zinserling, N.Y. Semenova, A.G. Markov, O.V. Rybalchenko, J. Wang, R.N. Rodionov et al. Inflammatory cell infiltration of adrenals in COVID-19. Horm. Metab. Res. 52(09), 639-641 (2020)

62. K.J. Ioakim, G.I. Sydney, S.A. Paschou, Glucose metabolism disorders in patients with adrenal gland disorders: pathophysiology and management. Hormones 19(2), 135-143 (2020)

63. O.J. McElvaney, N. McEvoy, O.F. McElvaney, T.P. Carroll, M.P. Murphy, D.M. Dunlea et al. Characterization of the inflammatory response to severe COVID-19 illness. Am. J. Respir. Crit. Care Med. 202, 812-821 (2020)

64. M. Migaud, S. Gandotra, H.S. Chand, M.N. Gillespie, V.J. Thannickal, R.J. Langley, Metabolomics to predict antiviral drug efficacy in COVID-19. Am. J. Respiratory Cell Mol. Biol. 63(3), 396-398 (2020)

65. H.A. Laviada-Molina, I. Leal-Berumen, E. Rodriguez-Ayala, R.A. Bastarrachea, Working hypothesis for glucose metabolism and SARS-CoV-2 replication: interplay between the hexosamine pathway and interferon RF5 triggering hyperinflammation. Role of BCG vaccine? Front. Endocrinol. 11, 514 (2020)

66. J. Mori, G. Y. Oudit, G. D. Lopaschuk, SARS-CoV-2 perturbs the Renin-Angiotensin System and energy metabolism. Am. J. Physiol.-Endocrinol. Metab. 319, E43-E47 (2020)

67. E. Eshak, A. Arafa, Thiamine deficiency and cardiovascular disorders. Nutr., Metab. Cardiovasc Dis. 28(10), 965-972 (2018) 
68. M. Çiftçi, İ. Özmen, M.E. Büyükokuroğlu, S. Pençe, Küfrevioğlu Öİ. Effects of metamizol and magnesium sulfate on enzyme activity of glucose 6-phosphate dehydrogenase from human erythrocyte in vitro and rat erythrocyte in vivo. Clin. Biochem. 34(4), 297-302 (2001)

69. S.F. Nabavi, S. Habtemariam, A. Sureda, M. Banach, I. BerindanNeagoe, C.A. Cismaru et al. Glucose-6-phosphate dehydrogenase deficiency and SARS-CoV-2 mortality: Is there a link and what should we do? Clin. Biochem. 86, 31-33 (2020)

70. R.J. Reiter, R. Sharma, Q. Ma, C. Liu, W. Manucha, P. AbreuGonzalez et al. Plasticity of glucose metabolism in activated immune cells: advantages for melatonin inhibition of COVID-19 disease. Melatonin Res. 3(3), 362-379 (2020)

71. M.A. El-Missiry, Z.M. El-Missiry, Melatonin is a potential adjuvant to improve clinical outcomes in individuals with obesity and diabetes with coexistence of Covid-19. Eur. J. Pharmacol. 882, 173329 (2020)
72. Q. Ye, B. Wang, J. Mao, Cytokine storm in COVID-19 and treatment. J. Infect. 80(6), 607-613 (2020)

73. G. Anderson, R.J. Reiter, Melatonin: roles in influenza, Covid-19, and other viral infections. Rev. Med. Virol. 30(3), e2109 (2020)

74. L. Barrea, G. Pugliese, L. Framondi, R. Di Matteo, D. Laudisio, S. Savastano, et al. Does Sars-Cov-2 threaten our dreams? Effect of quarantine on sleep quality and body mass index. J Transl Med. 18, 318 (2020)

75. L. Luzi, M.G. Radaelli, Influenza and obesity: its odd relationship and the lessons for COVID-19 pandemic. Acta Diabetol. 57, 759-764 (2020)

76. G. Tannahill, A. Curtis, J. Adamik, E. Palsson-McDermott, A. McGettrick, G. Goel et al. Succinate is an inflammatory signal that induces IL-1 $\beta$ through HIF-1 $\alpha$. Nature 496(7444), 238-242 (2013)

77. E. Akkus, M. Sahin, Related molecular mechanisms of COVID19, hypertension, and diabetes. Am. J. Physiol.-Endocrinol. Metab. 318(6), E881-E881 (2020) 